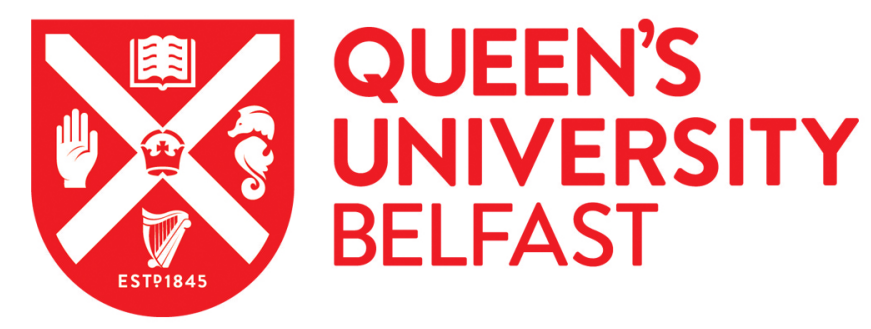

\title{
A Quantitative Analysis of the Role of Oxygen Tension in FLASH Radiation Therapy
}

Petersson, K., Adrian, G., Butterworth, K., \& McMahon, S. J. (2020). A Quantitative Analysis of the Role of Oxygen Tension in FLASH Radiation Therapy. International Journal of Radiation Oncology Biology Physics. https://doi.org/10.1016/j.ijrobp.2020.02.634

Published in:

International Journal of Radiation Oncology Biology Physics

Document Version:

Peer reviewed version

Queen's University Belfast - Research Portal:

Link to publication record in Queen's University Belfast Research Portal

Publisher rights

Copyright 2020 Elsevier Ltd.

This manuscript is distributed under a Creative Commons Attribution-NonCommercial-NoDerivs License

(https://creativecommons.org/licenses/by-nc-nd/4.0/), which permits distribution and reproduction for non-commercial purposes, provided the author and source are cited

\section{General rights}

Copyright for the publications made accessible via the Queen's University Belfast Research Portal is retained by the author(s) and / or other copyright owners and it is a condition of accessing these publications that users recognise and abide by the legal requirements associated with these rights.

Take down policy

The Research Portal is Queen's institutional repository that provides access to Queen's research output. Every effort has been made to ensure that content in the Research Portal does not infringe any person's rights, or applicable UK laws. If you discover content in the Research Portal that you believe breaches copyright or violates any law, please contact openaccess@qub.ac.uk. 


\section{A quantitative analysis of the role of oxygen tension in FLASH radiotherapy}

Kristoffer Petersson, $\mathrm{PhD}^{1,2}$, Gabriel Adrian, $\mathrm{MD}^{3}$, Karl Butterworth $\mathrm{PhD}^{4}$, Stephen J McMahon PhD ${ }^{4, *,+}$

1. The Oxford Institute for Radiation Oncology, Department of Oncology, University of Oxford, Oxford, UK.

2. Radiation Physics, Department of Haematology, Oncology and Radiation Physics, Skåne University Hospital, Lund, Sweden.

3. Department of Clinical Sciences Lund, Oncology, Skåne University Hospital, Lund University, Lund, Sweden

4. Centre for Cancer Research and Cell Biology, Queen's University Belfast, Belfast, Northern Ireland

† - Author responsible for statistical analysis

* - Author to whom correspondence should be addressed:

Stephen.mcmahon@qub.ac.uk

Centre for Cancer Research and Cell Biology

Queen's University Belfast

97 Lisburn Road

Belfast

BT9 7AE

Northern Ireland

+44 (0)2890972620

\section{Final version}

This is a preprint copy of a manuscript and supplementary material, accepted at the International Journal of Radiation Oncology, Biology, Physics. It is available online at:

https://doi.org/10.1016/j.ijrobp.2020.02.634

https://www.sciencedirect.com/science/article/pii/S0360301620308841 


\section{Abstract}

\section{Background}

Recent demonstrations of normal tissue sparing by high dose, high dose rate FLASH radiotherapy have driven considerable interest in its application to improve clinical outcomes. However, there remains significant uncertainty about the underlying mechanisms of FLASH sparing, and how deliveries can be optimised to maximize benefit from this effect. Rapid oxygen depletion has been suggested as a potential mechanism by which these effects occur, but has yet to be quantitatively tested against experimental data.

\section{Methods}

Models of oxygen kinetics during irradiation were used to develop a time-dependent model of the Oxygen Enhancement Ratio (OER) in mammalian cells that incorporates oxygen depletion. The characteristics of this model were then explored in terms of the dose- and dose rate dependence of the OER. This model was also fit to experimental data from both in vitro and in vivo datasets.

\section{Results}

In cases of FLASH radiotherapy, this model suggests that oxygen levels can be depleted by amounts which are sufficient to impact on radiosensitivity only in conditions of intermediate oxygen tension, with no effect seen at high or very low oxygen levels. The model also effectively reproduced the dose, dose rate and oxygen tension dependence of responses to FLASH radiotherapy in a range of systems, with model parameters compatible with published data.

\section{Conclusions}

Oxygen depletion provides a credible quantitative model to understand the biological effects of FLASH radiotherapy and is compatible with a range of experimental observations of FLASH sparing. These results highlight the need for more detailed quantification of oxygen depletion under high dose rate radiation exposures in relevant systems, and the importance of oxygen tension in target tissues for FLASH sparing to be observed. 


\section{Introduction}

The tissue-sparing effects of FLASH radiotherapy - large doses of radiation delivered at extremely high dose rates (tens of Gray per second or more) - have been the subject of considerable interest following recent experimental data showing that it can effectively preserve normal tissues while retaining antitumour efficacy $(1,2)$. The exact mechanism of the FLASH effect is subject to debate, as the dose rates where the effect is observed are significantly faster than those associated with radiobiological processes such as repair and repopulation, but still orders of magnitude slower than those where inter-track physical interactions may have an impact.

As a result, a major topic of interest has been the impact of FLASH exposures on radiation chemistry, in particular involving the depletion of oxygen. Oxygen plays a significant role in determining radiosensitivity, through the Oxygen Enhancement Ratio (OER) (3-8), where well-oxygenated cells are more sensitive to ionising radiation than fully hypoxic cells, by approximately a factor of three. Under normal circumstances, oxygen tension does not vary significantly during radiotherapy as the rate of oxygen degradation is significantly slower than that of oxygen replenishment from the surrounding environment.

However, significant research over the past fifty years in bacteria, mammalian cell lines, and small animal models has demonstrated that, at very high dose rates, depletion of oxygen can lead to significant sparing (9-17). Despite these early results, ultra-high dose rate irradiation was not translated into a clinical treatment technique. However, greater availability of ultra-high dose rate irradiation systems together with a number of published studies demonstrating FLASH effects have renewed interest in this field (18-23), with several groups suggesting radiation-induced oxygen depletion as a potential mechanism.

Significant questions remain regarding the implications of this model on the underlying features of the FLASH effect. These include if it has thresholds for dose, dose rate or total delivery time, and how it delivers a differential toxicity between normal and tumour tissues.

In this work, we update models of time-varying OER from previous literature to describe how they can be applied in mammalian cells, and fit them to both in vitro and in vivo datasets. The predictive power of the model was evaluated to provide initial estimates of key radiation response parameters including the oxygen depletion and recovery rates in biological systems. Finally, we discuss the implications of this model for potential clinical exploitation of the FLASH effect.

\section{Methods}

\section{Oxygen Kinetics}

The kinetics of oxygen under high-dose and high dose rate irradiation have previously been studied in a number of systems, and are used as the basis of a simplified model of $\mathrm{O}_{2}$ availability. A number of models of oxygen kinetics in irradiated systems have been proposed, including depletion which is linear in dose, or through first- or second-order reactions with radiation products $(12,14,16,24)$. For exposures carried out in pure water or dilute solutions, experimental measurements and computational models of dissolved oxygen suggest oxygen depletion is independent of $\mathrm{O}_{2}$ concentration down to very low concentrations $(14,25)$. However, within cells and tissues, a large number of other molecules are present which can interact with radiolysis products, potentially altering these kinetics by introducing competition for reactions with dissolved oxygen.

For this analysis, we investigated both first-order and concentration independent interaction rates between radiation and dissolved oxygen. The first-order model is presented below, with the concentration independent model presented in the Supplementary Information. The first-order 
interaction rate is defined in terms of oxygen depletion scaling with the dose rate and current oxygen concentration. That is:

$$
\frac{d O}{d t}=-g \dot{D} O
$$

Where $O$ is the current oxygen concentration, $\dot{D}$ is the dose rate, and $g$ is the depletion rate constant which takes units of $\mathrm{Gy}^{-1}$.

This oxygen depletion is in competition with reoxygenation (from the surrounding atmosphere in cell culture, or nearby blood vessels in vivo). In the in vitro case, cells are contained in media which can be reoxygenated through gas exchange with the surrounding air, which can be approximated as a firstorder diffusion process depending on the difference in oxygen concentrations (14), that is:

$$
\frac{d O}{d t}=\lambda\left(O_{e n v}-O\right)
$$

Where $O_{\text {env }}$ is the (micro-) environmental oxygen tension in the absence of irradiation and $\lambda$ is the oxygen recovery rate. A fully detailed model of in vivo oxygen diffusion would necessarily be very complex, having to account for parameters such as blood flow, haemoglobin level, diffusion of oxygen, and any potential damage to blood vessels by irradiation. As a first approximation, we have considered applying the same first-order kinetics to determine how well this simplest model can reproduce in vivo observations.

Equations 2 and 3 can be combined into a single expression describing the behaviour of oxygen under irradiation, as:

$$
\frac{d O}{d t}=-g \dot{D} O+\lambda\left(O_{e n v}-O\right)=-(g \dot{D}+\lambda) O+\lambda O_{e n v}=-\gamma O+\lambda O_{e n v}
$$

Here, $\gamma=g \dot{D}+\lambda$ has been introduced as a simplifying term to represent the rate of change in $\mathrm{O}_{2}$ concentration when exposed at a particular dose rate. This expression can be solved to give:

$$
O(t)=O_{e n v}\left(\frac{\lambda}{\gamma}+\left(1-\frac{\lambda}{\gamma}\right) e^{-\gamma t}\right)
$$

That is, the oxygen level begins at the environmental concentration $O_{\text {env }}$, but falls exponentially with radiation exposure until it has been reduced by a factor of $\lambda / \gamma$. Note this ratio is approximately 1 when the dose rate is small, but falls to zero as the dose rate increases.

These parameters can be related to the delivered dose and dose rate in a number of ways. For example, a 'characteristic dose' can be defined as the dose which reduces the oxygen concentration to $1 / e$ of its initial value in the absence of recovery, that is: $D_{c}=\frac{1}{g}$, where $D_{c}$ is the characteristic dose. Similarly, a 'characteristic dose rate' of a system can be defined as that needed to give a significant reduction in the oxygen concentration at equilibrium. If a threshold of $50 \%$ is set, then this can be shown to be $\dot{D}_{c}=\frac{\lambda}{g}$, where $\dot{D}_{c}$ is the characteristic dose rate. As will be seen below, for significant FLASH effects to be seen, both the delivered dose and delivered dose rate should be greater than $D_{c}$ and $\dot{D}_{c}$, respectively.

Oxygen tensions are reported in a number of ways, including as $\mathrm{O} 2$ concentration as a fraction of atmospheric pressure, or partial pressures in terms of $\mathrm{mm}$ of mercury $(\mathrm{mmHg})$. In this work, oxygen concentrations have been reported in these units based on a pressure of 1 atmosphere and temperature of $37 \mathrm{C}$. In principle, these values can also be converted into molar concentrations of 
oxygen (i.e. Moles/L), but this requires knowledge of the solubility of oxygen in these systems, which remains poorly determined (26).

\section{Oxygen Enhancement Ratio}

OERs are typically specified as a simple sigmoid function of the environmental oxygen level. A number of parameterisations are used, with among the most common being $(4,27)$ :

$$
O E R=\frac{K+m O_{e n v}}{K+O_{e n v}}
$$

Where $m$ is the maximum OER (typically $\approx 3$ ) and $K$ is the oxygen tension at the midpoint of the OER curve. However, this assumes that the oxygen level does not vary significantly throughout the exposure. If it does, we must instead consider how the OER varies as a function of time, and integrate this throughout the irradiation to generate an averaged OER. Specifically, the averaged OER for a dose delivered at a constant rate of $\dot{D}$ for a time $T$ can be expressed as:

$$
\overline{O E R}=\frac{1}{T} \int_{0}^{T} \frac{K+m O(t)}{K+O(t)} d t
$$

Where $\mathrm{T}$ is the total time of the irradiation, and $O(t)$ is the time-varying oxygen concentration as defined above. This approach assumes that damage caused by irradiation is determined by the oxygen concentration at the moment it is delivered. In reality, experiments investigating the impact of reoxygenation showed that damage is fixed by reaction with $\mathrm{O}_{2}$ over a very short period immediately following irradiation (28). Fixing of damage was seen to occur with a half-life of around $500 \mu \mathrm{s}$ in bacteria (29), and was seen to be largely complete within $5 \mathrm{~ms}$ in mammalian cells (30). In practice, this would act to slightly modify the OER for the most rapid exposures, but this effect is typically small as reoxygenation kinetics are believed to happen over much longer timescales.

Equation 6 can be integrated to give a (somewhat unwieldly) analytic expression for the averaged time-varying OER of:

$$
\overline{O E R}=3-\frac{2 K}{T\left(\gamma K+\lambda O_{e n v}\right)} \log \left(\frac{(\gamma-\lambda) O_{e n v}+\left(\gamma K+\lambda O_{e n v}\right) \mathrm{e}^{\gamma T}}{\gamma\left(K+O_{e n v}\right)}\right)
$$

Here, we have fixed the maximum OER of $m=3$ for simplicity. It can be seen that when $T$ is small or the rate of change parameter $\gamma \approx \lambda$ (that is, the depletion rate $g \dot{D}$ is much smaller than $\lambda$ ), the average OER approaches the constant OER for environmental oxygen levels. At high dose rates and times, it instead approaches the OER for an oxygen level of $O_{e n v} \lambda / \gamma$, the equilibrium concentration under irradiation. The exact value of $\overline{O E R}$ is a complex function depending on the interplay between the initial oxygen level, the dose rate, and the total dose delivered (via the exposure time $T$ ).

\section{In vitro analysis}

To test this model's predictive power, it was fit to a dataset investigating the clonogenic sensitivity of DU-145 prostate cancer cells to either conventional (14 Gy/min) or FLASH (600 Gy/s) 10 MeV electron irradiation using a modified Elekta Precise Linac (31) at a range of oxygen tensions (32). This includes full dose-response curves under oxic conditions (20\% atmospheric content, equivalent to $150 \mathrm{mmHg}$ ) and hypoxia $\left(1.6 \% \mathrm{O}_{2}, 12 \mathrm{mmHg}\right)$ and a series of exposures to $18 \mathrm{~Gy}$ at different oxygen tensions. All of the data was included in a weighted nonlinear regression fit, using the standard LQ model of cellular response to predict survival under each condition, and accounting for varying OER through oxygen depletion using equation 7 above. 
A total of four parameters were fit to this data set: the LQ parameters $\alpha$ and $\beta$ for response under oxic conditions at conventional dose rates, the oxygen depletion rate $g$ and the mid-point of the OER curve, $K$. Due to limitations in the dataset it was not possible to fit the recovery rate of oxygen explicitly, so $\lambda$ has been fixed at a constant value of $1 \mathrm{~s}^{-1}$.

In vivo analysis

To investigate the model's predictive power in an in vivo model, the time-varying OER was applied to published data on neuroprotective effects following FLASH whole-brain irradiation (2). In this study, mice were irradiated to a dose of $10 \mathrm{~Gy}$ at a range of different dose rates using customised 4.5 or 6 $\mathrm{MeV}$ electron Linacs. After two months, their neural function was evaluated in the Novel Object Recognition test, testing their preference for investigating novel objects.

Effective delivered doses were calculated using the time-varying OER above, varying the oxygen recovery rate $\lambda$. As corresponding dose response curves were not available, OER parameters were matched to the in vitro analysis $\left(K=1.0 \% \mathrm{O}_{2}, m=3\right)$, and the magnitude of response was modelled as a simple linear function of dose, that is $\Delta R R=1-\frac{\overline{O E R} \times D}{D_{0}}$, where $\Delta R R$ is the change in recognition ratio, $D$ is the physical dose delivered which is scaled by the average oxygen enhancement ratio $\overline{O E R}$, and $D_{0}$ is a rate defining the dose response of this test. For this analysis, it was assumed that the oxygen concentration in the brain tissue was $3.4 \%(26 \mathrm{mmHg})$, as reported in previous studies (33).

\section{Alternative Oxygen Kinetics Models}

As noted above, the exact kinetics of oxygen depletion and recovery remain somewhat uncertain. To test if alternative oxygen depletion models qualitatively impacted on these predictions, $\overline{O E R}$ was also simulated under the assumption that the depletion of $\mathrm{O}_{2}$ is directly proportional to the delivered dose, independent of oxygen concentration. Results on this analysis are presented in the Supplementary Information.

Results

\section{Oxygen Kinetics}

The variation of oxygen under different irradiation conditions can be simulated by applying this oxygen kinetic model. These rates are illustrated in Figure 1 , for a region initially containing $\mathrm{O}_{2}$ at $5 \%$ of atmospheric concentration ( $38 \mathrm{mmHg}$ ), exposed to dose rates ranging from 0.1 to $100 \mathrm{~Gy} / \mathrm{s}$. Here, the critical dose has been set to $10 \mathrm{~Gy}$ and the dose rate to $30 \mathrm{~Gy} / \mathrm{s}$, corresponding to $g=0.1 \mathrm{~Gy}^{-1}$ and $\lambda=3 \mathrm{~s}^{-1}$.

Irradiation at low dose rates (where $g \dot{D}$ is much smaller than $\lambda$ ) shows negligible impacts on oxygen levels, but more rapid deliveries are associated with more significant depletion of oxygen. At very high dose rates, this can lead to the removal of the majority of free oxygen in less than a second, potentially resulting in significant changes in the average oxygen levels seen during FLASH exposures.

Figure $1 \mathrm{~b}$ presents how these effects depend on the delivered dose. For all dose rates, the initial slope of oxygen depletion with dose is identical (proportional to the delivered dose times $g$ ), but the curves plateau at different levels as oxygen recovery becomes significant. Thus, combinations of both high doses and high dose rates are needed to significantly deplete oxygen in a way which will impact on OER.

\section{Average Oxygen Enhancement Ratio}

Figure 2 shows the relationship between environmental oxygen levels and $\overline{O E R}$ for different irradiation conditions. At low doses and dose rates, the model predicts that $\overline{O E R}$ is equal to 
conventional OER with an oxygen concentration of $O_{\text {env }}$. Similarly, at high oxygen concentrations, even relatively large doses do not substantially deplete the available oxygen and there is no significant impact on $\overline{O E R}$.

However, as oxygen levels are reduced (to $1-5 \%$ of an atmosphere, $5-40 \mathrm{mmHg}$ ) oxygen depletion can become a significant factor, leading to a lower $\overline{O E R}$ than predicted from the initial $\mathrm{O}_{2}$ concentration. This is potentially biologically relevant as many cells in normal tissues are at this intermediate oxygen level, termed 'physoxia' (33). Significantly, at these intermediate oxygen levels the effect depends on the dose rate (Figure $2 \mathrm{a}$ ) and the total dose (Figure $2 \mathrm{~b}$ ), which both have to be sufficiently large to deliver significant FLASH-related sparing. Finally, at lower oxygen concentrations, these curves begin to converge once again as the depletion of oxygen in cells beginning at extremely level of $\mathrm{O}_{2}$ does not substantially impact on overall sensitivity (7).

These overall trends are shared between any oxygen depletion and recovery parameters, with the values determining the thresholds at which FLASH effects are observed, and the degree of protection they offer. While these curves qualitatively agree with experimental observations of FLASH radiotherapy, fitting to experimental data is required to quantitatively characterise model parameters

In vitro analysis

Figure 3 presents an analysis of FLASH radioprotection in DU145 cells. As previously reported, these results show no statistically significant difference is seen between FLASH and conventional exposures at high oxygen levels (extra sum-of-squares F-test, $p=0.16$ ), while significant differences are seen between dose rates under hypoxia (extra sum-of-squares F-test, $p=0.013$ ). Moreover, this figure shows that the oxygen depletion model (lines) can effectively reproduce observed trends with dose, dose rate and oxygen tension.

In particular, the model accurately reflects the fact that there is negligible difference between the responses to both modalities in oxic conditions, while showing that they begin to separate at higher doses in hypoxic conditions due to oxygen depletion (Figure 3a). It also reproduces the experimental OER curves as a function of oxygen concentration for conventional and FLASH dose rates (Figure 3b). The best-fitting parameters were $\alpha=0.12 \pm 0.03 \mathrm{~Gy}^{-1}, \beta=0.027 \pm 0.002 \mathrm{~Gy}^{-2}, g=0.053 \pm 0.01$ $\mathrm{Gy}^{-1}$ and $K=1 \pm 0.1 \% \mathrm{O}_{2}(7.6 \mathrm{mmHg})$ for fixed parameters of $\lambda=1 \mathrm{~s}^{-1}$ and $m=3$.

These results correspond to a characteristic dose of approximately $18 \mathrm{~Gy}$, and a characteristic dose rate of $18 \mathrm{~Gy} / \mathrm{s}$. However, as only very low and high dose rates are represented in this dataset, these fits do not depend strongly on the value for $\lambda$, with recovery rates from 0.1 to $20 \mathrm{~s}^{-1}$ giving approximately the same fitting results (corresponding to characteristic dose rates between 2 and 400 $\mathrm{Gy} / \mathrm{s})$.

\section{In vivo analysis}

Figure 4 compares the variation in the Novel Object Recognition ratio to the variation in $\overline{O E R}$ at a range of dose rates, providing an estimation of the dose rate response in this system. Once again, the oxygen depletion model is able to effectively reproduce the dose rate dependence of these effects, with a best-fitting oxygen recovery rate of $\lambda=7.9 \pm 4 s^{-1}$. This data clearly shows the onset of the FLASH effect as the dose rate becomes similar in magnitude to the recovery rate, and saturation at higher dose rates when the dose is delivered in times much shorter than the oxygen recovery rate, as observed experimentally.

As reference dose-response curves are not available for this system, it is not possible in this analysis to explicitly determine the oxygen depletion rate $g$. As a result, the modelled dose is currently plotted in arbitrary units. However, as it can be seen that $10 \mathrm{~Gy}$ delivers a significant FLASH effect in this 
system, it suggests that the oxygen depletion rate is somewhat higher than that observed in the in vitro system, potentially indicating the influence of chain reactions which amplify the depletion of oxygen in the system, or the involvement of other tissue-specific effects (18). Possible implications for $g$ in vivo are discussed in more detail below.

\section{Discussion}

The ability of radiation delivered at high dose rates to deplete available oxygen and induce sparing is not a new concept, with results from past decades providing substantial evidence of these effects in bacterial $(12,14)$ and small animal models $(15)$. Here, we have revisited these models in the context of recent reports of FLASH radiotherapy sparing, and demonstrated that they are able to effectively reproduce many of the observed trends seen in experimental investigations of this effect.

This model is deliberately simplified, implementing the minimal possible mechanisms of oxygen depletion and recovery, together with an OER-dependent protection mechanism. In reality, neither oxygen depletion nor recovery are likely to be driven through single mechanisms as presented here. Higher-order contributions from processes such as the dismutation of superoxides or radical-radical recombinations in lipids may alter the overall kinetics of oxygen in irradiated systems (18). Similarly, there may be other, currently unaccounted-for, differences in the types of genetic damage caused at ultra-high dose rates, which cannot be definitively ruled out in the current analysis. However, the fact that the model reproduces observed trends in a range of systems suggests that these higher-order processes are not a major contribution to the FLASH effect, and may be difficult to distinguish from dose and dose rate studies alone.

From this model, we can make the following observations about the effects of oxygen depletion in FLASH:

i) The FLASH effect depends on a combination of both high dose and dose rate. Even in the absence of oxygen recovery, sufficient dose is needed to deplete available oxygen, as highlighted by the gradual separation of the hypoxic curves in Figure 3a at higher doses. Alongside this, sufficiently high dose rates are necessary to deplete available oxygen more rapidly than it can be recovered. The exact dose- and dose rate thresholds depend on the kinetics of oxygen within the system.

ii) It is the total dose rate across the entire exposure, rather than the instantaneous dose rate, which is of particular relevance in driving oxygen depletion relevant to FLASH effects. No significant difference is seen in response between high doses rates delivered continuously and in a series of pulses (see Supplementary Information). In cases with a high instantaneous dose rate but low total dose rate (such as delivery of radiation in pulses from a conventional Linac), recovery between pulses will mitigate any oxygen depletion within pulses and prevent FLASH sparing. This may pose a challenge for modern, modulated radiotherapy approaches, where treatments comprising multiple fields whose delivery is separated by even a second may completely abrogate the benefits of high dose rates.

iii) FLASH effects are not expected to be observed at atmospheric $\mathrm{O}_{2}$ levels. Even for instantaneous deliveries, the doses needed to deplete $20 \%$ oxygen to hypoxic conditions are on the order of tens to hundreds of Gray, This is far outside a practical radiobiological or therapeutic range, and may explain the absence of effect seen in some in vitro tests at high dose rates (34). In vivo sparing is observed because most tissues are at an intermediate oxygen tension, termed 'physoxia', with median oxygenation levels ranging from $3-7 \% \mathrm{O}_{2}(20-50 \mathrm{mmHg})(33)$. At this level, oxygen depletion becomes practical and contributes to radioresistance. This is supported by recent data which show that artificially increasing the oxygen tension in tissues mitigates FLASH sparing (20). 
iv) Preservation of anti-tumour efficacy in vivo may be driven by the hypoxic core commonly seen in xenograft tumour models. Many tumours have a median oxygen tension less than $1 \%(7.6 \mathrm{mmHg})$, and almost all xenograft tumours have a significant fraction of fully hypoxic cells with negligible remaining oxygen $(33,35)$. These resistant hypoxic cells are believed to represent the majority of cells which survive irradiation and lead to tumour recurrence. As a result, even though FLASH sparing can be observed in cancer cells (as shown in Figure 3 (32)), depletion of any remaining oxygen in this highly hypoxic core does not significantly impact on observed tumour sensitivity.

These results provide potential guidance for the use of FLASH radiotherapy in the clinic. This model supports current experimental observations that FLASH is an effect of both high doses and high dose rates, centrally driven by oxygen depletion. In addition, it can be seen that these overall trends do not strongly depend on the chosen model of oxygen depletion, with similar effects seen when alternative models are considered (see Supplementary Information). Further data is needed to statistically distinguish between different oxygen depletion models, and this analysis raises a number of questions critical for optimising FLASH delivery.

Many of the key response parameters remain quite uncertain, particularly oxygen depletion and recovery rates, which makes it difficult to distinguish between possible models. At present, there are insufficient datasets available to directly experimentally determine or robustly fit all of these values for a single well-defined condition. It is known that the rate of oxygen depletion with radiation can strongly depend on the medium and cellular conditions (14). A broad range of estimates have been proposed for potential oxygen depletion rates, ranging from $0.04 / G y$ in simple media (14) to more than 0.5 /Gy in cells when accounting for more complex radiochemical processes (18). These depletion rates would correspond to a broad range of predicted degrees of FLASH sparing. For example, for 10 Gy delivered to the brain, this could range from approximately $10 \%$ sparing predicted by the oxygen depletion rate obtained in our fit to in vitro data, to a reduction in effective dose by nearly a factor of 2 for the highest rates of depletion proposed in the literature (18).

While reported FLASH sparing effects fall within this range $(36,37)$ the exact magnitude of the effect remains uncertain, and is potentially strongly condition-dependent. Early work suggested potential sparing effects equivalent to almost a four-fold reduction in dose, which could not be easily explained by hypoxia alone (1), while more recent studies have suggested protection factors on the order of 1.41.8 , which are more compatible with hypoxia as a driving mechanism (36). Further experiments designed and powered to accurately determine both dose- and dose rate dependence alongside any potential tissue-type specificity will be an important step in better understanding the role of oxygen depletion FLASH radiotherapy.

Experiments specifically targeting particular aspects of radical biology in cells, such as through altering scavenging and processing of superoxides, would also prove valuable in quantifying precisely which processes are the key drivers of FLASH radiobiology. Similarly, a better understanding of the kinetics of reoxygenation in tissues following high dose rate radiotherapy would be necessary to fully understand FLASH effects in tissues. While a first-order approximation appears to capture the majority of the variation in in vivo observations (Figure 4), the true behaviour is likely significantly more complex, and there may be significant benefit in mapping out how these effects depend on local tissue and vasculature architecture (38-40).

Finally, this model strongly suggests that the local oxygen availability is a key factor in the magnitude of the effect of FLASH radiotherapy, with significant sparing seen at intermediate oxygen tensions, but little at either very high or very low oxygen levels. While this effectively preserves efficacy in xenograft tumours which have substantial hypoxic fractions, it is possible that some better-oxygenated tumours 
which are encountered clinically may see some unexpected sparing. As a result, careful consideration of tumour oxygenation may be important factor in early clinical applications of FLASH.

\section{Conclusions}

Models of radiation-induced oxygen depletion effectively reproduce the kinetics of FLASH radiotherapy observed in in vitro and in vivo endpoints. This provides an explanation for many of the observed trends in the FLASH effect, but also highlights significant uncertainties which remain in quantifying its benefit for clinical applications.

\section{References}

1. Favaudon V, Caplier L, Monceau V, et al. Ultrahigh dose-rate FLASH irradiation increases the differential response between normal and tumor tissue in mice. Sci. Transl. Med. 2014;6:245ra93.

2. Montay-Gruel $\mathrm{P}$, Petersson $\mathrm{K}$, Jaccard $\mathrm{M}$, et al. Irradiation in a flash: Unique sparing of memory in mice after whole brain irradiation with dose rates above 100 Gy/s. Radiother. Oncol. 2017;124:365369.

3. GRAY LH, CONGER AD, EBERT M, et al. The concentration of oxygen dissolved in tissues at the time of irradiation as a factor in radiotherapy. Br. J. Radiol. 1953;26:638-648.

4. Alper T, Howard-Flanders P. Role of oxygen in modifying the radiosensitivity of E. coli B. Nature. 1956;178:978-979.

5. Hall EJ. The effect of hypoxia on the repair of sublethal radiation damage in cultured mammalian cells. Radiat. Res. 1972;49:405-415.

6. Chapman JD, Dugle DL, Reuvers AP, et al. Studies on the radiosensitizing effect of oxygen in chinese hamster cells. Int. J. Radiat. Biol. 1974;26:383-389.

7. Wouters BG, Brown JM. Cells at intermediate oxygen levels can be more important than the "hypoxic fraction" in determining tumor response to fractionated radiotherapy. Radiat. Res. 1997;147:541-550.

8. Grimes DR, Partridge M. A mechanistic investigation of the oxygen fixation hypothesis and oxygen enhancement ratio. Biomed. Phys. Eng. Express. 2015;1:45209.

9. Dewey DL, Boag JW. Modification of the oxygen effect when bacteria are given large pulses of radiation. Nature. 1959;183:1450-1451.

10. Town CD. Effect of High Dose Rates on Survival of Mammalian Cells. Nature. 1967;215:847-848.

11. Todd P, Winchell HS, Feola JM, et al. Pulsed high-intensity roentgen rays: Inactivation of human cells cultured in vitro and limitations on usefulness in radiotherapy. Acta Oncol. (Madr). 1968;7:2226.

12. Ling CC. Time Scale of Radiation-Induced Oxygen Depletion and Decay Kinetics of OxygenDependent Damage in Cells Irradiated at Ultrahigh Dose Rates. Radiat. Res. 1975;63:455.

13. Michaels HB, Epp ER, Ling CC, et al. Oxygen Sensitization of CHO Cells at Ultrahigh Dose Rates: Prelude to Oxygen Diffusion Studies. Radiat. Res. 1978;76:510.

14. Whillans DW, Rauth AM. An Experimental and Analytical Study of Oxygen Depletion in Stirred Cell Suspensions. Radiat. Res. 1980;84:97.

15. Hendry JH, Moore J V., Hodgson BW, et al. The Constant Low Oxygen Concentration in All the Target Cells for Mouse Tail Radionecrosis. Radiat. Res. 1982;92:172. 
16. Weiss H, Epp ER, Heslin JM, et al. Oxygen Depletion in Cells Irradiated at Ultra-high Dose-rates and at Conventional Dose-rates. Int. J. Radiat. Biol. 1974;26:17-29.

17. Epp ER, Weiss H, Santomasso A. The Oxygen Effect in Bacterial Cells Irradiated with High-Intensity Pulsed Electrons. Radiat. Res. 1968;34:320.

18. Spitz DR, Buettner GR, Petronek MS, et al. An integrated physico-chemical approach for explaining the differential impact of FLASH versus conventional dose rate irradiation on cancer and normal tissue responses. Radiother. Oncol. 2019;139:23-27.

19. Pratx G, Kapp DS. Ultra-high dose rate FLASH irradiation may spare hypoxic stem cell niches in normal tissues. Int. J. Radiat. Oncol. 2019.

20. Montay-Gruel P, Acharya MM, Petersson K, et al. Long-term neurocognitive benefits of FLASH radiotherapy driven by reduced reactive oxygen species. Proc. Natl. Acad. Sci. U. S. A. 2019;116.

21. Vozenin MC, De Fornel P, Petersson K, et al. The Advantage of FLASH Radiotherapy Confirmed in Mini-pig and Cat-cancer Patients. Clin. Cancer Res. 2019;25:35-42.

22. Beyreuther $E$, Brand $M$, Hans $S$, et al. Feasibility of proton FLASH effect tested by zebrafish embryo irradiation. Radiother. Oncol. 2019;139:46-50.

23. Montay-Gruel P, Bouchet A, Jaccard M, et al. X-rays can trigger the FLASH effect: Ultra-high doserate synchrotron light source prevents normal brain injury after whole brain irradiation in mice. Radiother. Oncol. 2018;129:582-588.

24. Michaels HB. Oxygen depletion in irradiated aqueous solutions containing electron affinic hypoxic cell radiosensitizers. Int. J. Radiat. Oncol. Biol. Phys. 1986;12:1055-1058.

25. Boscolo D, Krämer M, Fuss MC, et al. Impact of target oxygenation on the chemical track evolution of ion and electron radiation. Int. J. Mol. Sci. 2020;21.

26. Place TL, Domann FE, Case AJ. Limitations of oxygen delivery to cells in culture: An underappreciated problem in basic and translational research. Free Radic. Biol. Med. 2017;113:311322.

27. Alper T, Moore JL, Smith P. The Role of Dose Rate, Irradiation Technique, and LET in Determining Radiosensitivities at Low Oxygen Concentrations. Radiat. Res. 1967;32:780.

28. Howard-Flanders $P$, Moore D. The time interval after pulsed irradiation within which injury to bacteria can be modified by dissolved oxygen. I. A search for an effect of oxygen 0.02 second after pulsed irradiation. Radiat. Res. 1958;9:422-437.

29. Michael BD, Adams GE, Hewitt HB, et al. A posteffect of oxygen in irradiated bacteria: a submillisecond fast mixing study. Radiat. Res. 1973;54:239-251.

30. Watts ME, Maughan RL, Michael BD. Fast kinetics of the oxygen effect in irradiated mammalian cells. Int. J. Radiat. Biol. 1978;33:195-199.

31. Lempart M, Blad B, Adrian G, et al. Modifying a clinical linear accelerator for delivery of ultra-high dose rate irradiation. Radiother. Oncol. 2019;139:40-45.

32. Adrian G, Petersson K. The FLASH effect depends on oxygen concentration. Submitted. 2019.

33. McKeown SR. Defining normoxia, physoxia and hypoxia in tumours - Implications for treatment response. Br. J. Radiol. 2014;87:1-12.

34. Buonanno M, Grilj V, Brenner DJ. Biological effects in normal cells exposed to FLASH dose rate protons. Radiother. Oncol. 2019. 
35. Olive PL, Banáth JP, Durand RE. The Range of Oxygenation in SiHa Tumor Xenografts. Radiat. Res. 2002;158:159-166.

36. Bourhis J, Montay-Gruel P, Gonçalves Jorge P, et al. Clinical translation of FLASH radiotherapy: Why and how? Radiother. Oncol. 2019:4-10.

37. Wilson JD, Hammond EM, Higgins GS, et al. Ultra-High Dose Rate (FLASH) Radiotherapy: Silver Bullet or Fool's Gold? Front. Oncol. 2020;9:1-12.

38. Cucinotta F, Cacao E, Hill M, et al. Modelling normal tissue injuiry in flash radiotherapy. In: Radiation Research Society Annual Meeting.; 2019:PS8-24.

39. Rothwell B, Merchant MJ, Chadwick AL, et al. An investigation into the feasibility of oxygen depletion as the mechanism behind FLASH. In: PTCOG58.; 2019:PTC58-0578.

40. Wilson $\mathrm{P}$, Jones $\mathrm{B}$, Yokoi $\mathrm{T}$, et al. Revisiting the ultra-high dose rate effect: Implications for charged particle radiotherapy using protons and light ions. Br. J. Radiol. 2012;85:933-939. 
Figures

a)

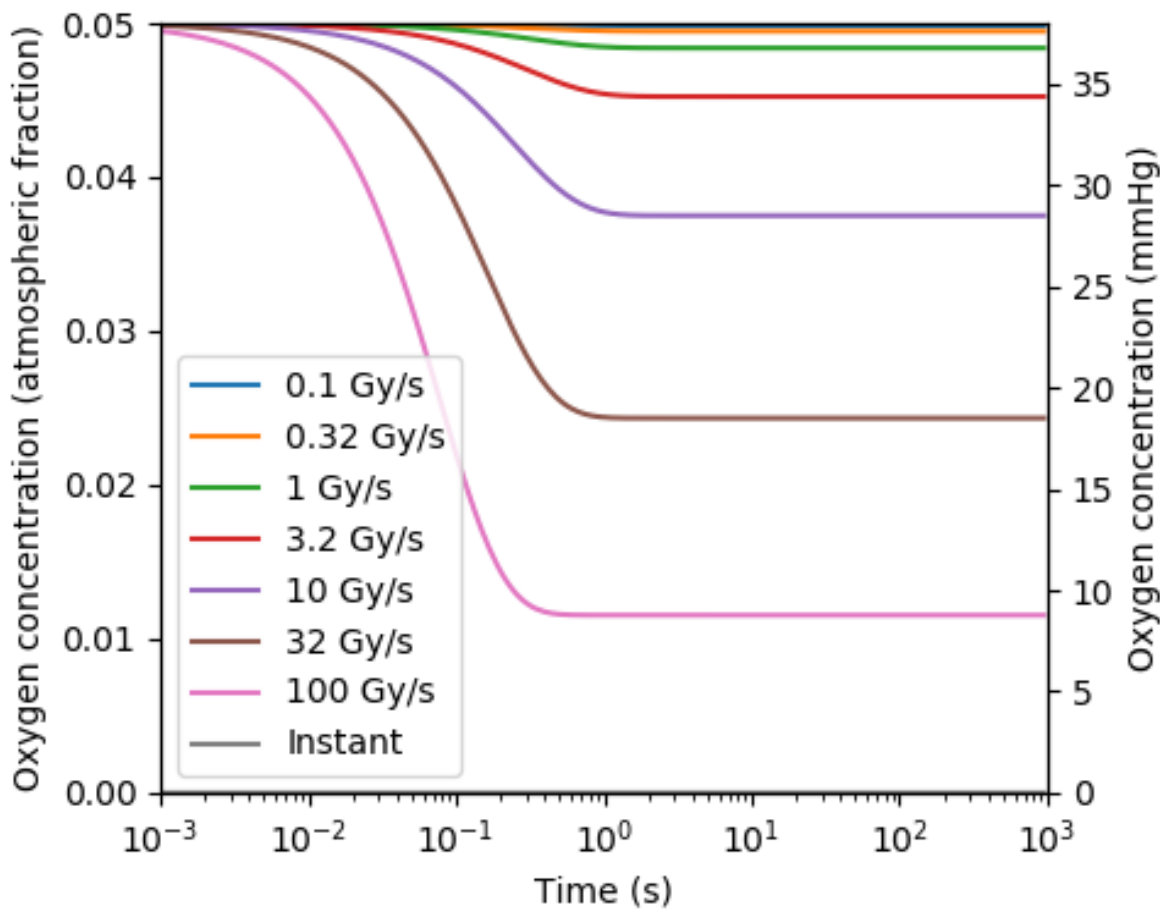

b)

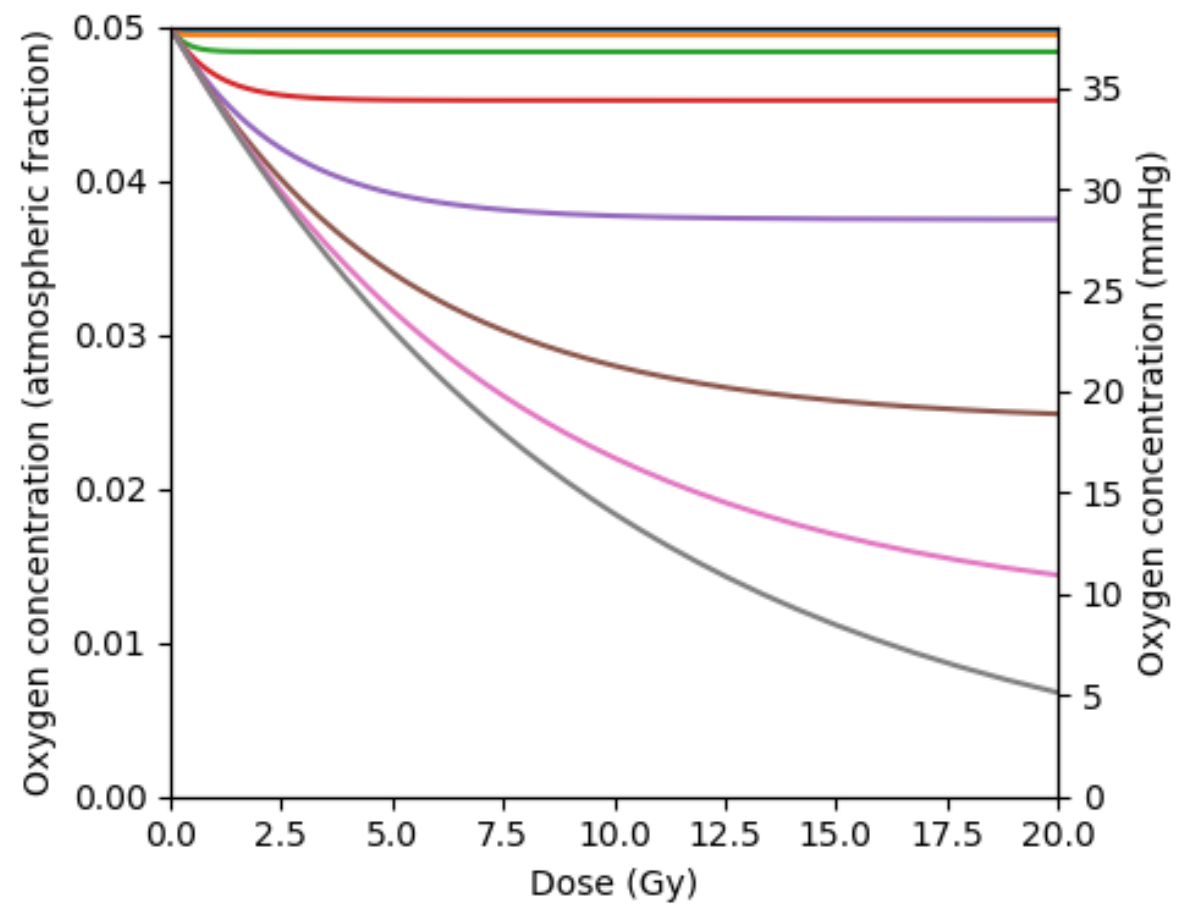

Figure 1: High dose rate irradiation can significantly deplete the available oxygen in an irradiated system. a) Oxygen variation as a function of irradiation time. While low dose rates have only a minor effect on oxygen levels, higher dose rates can rapidly lead to significant depletion of oxygen in these systems. b) Oxygen concentration as a function of dose. Irradiations at different dose rates tend to see initially similar reductions in $\mathrm{O} 2$ levels, but plateau at different points based on the relationship between oxygen depletion and recovery. For instantaneous dose-delivery this becomes a simple exponential function of dose. 
a)

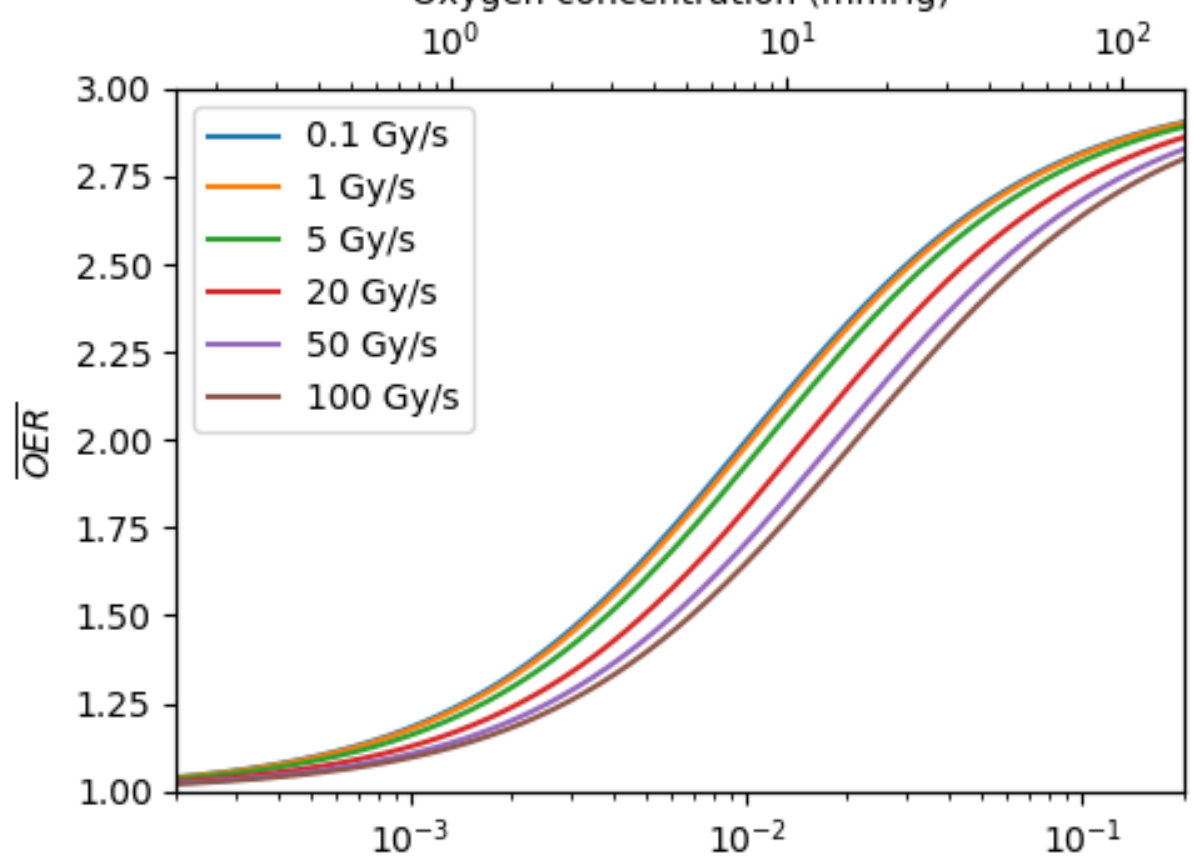

b)

$\mathrm{O}_{2}$ Concentration (atmospheric fraction)

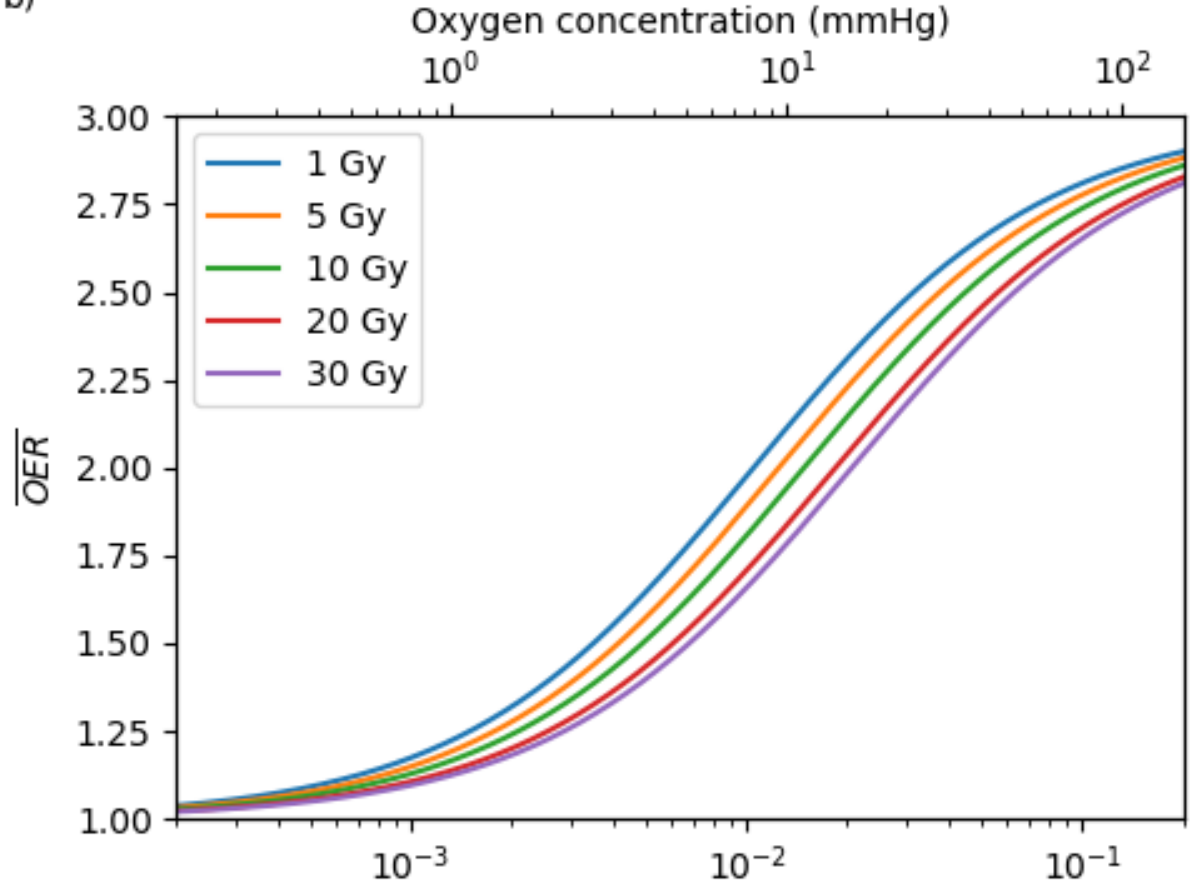

$\mathrm{O}_{2}$ Concentration (atmospheric fraction)

Figure 2: Variations in oxygen depletion can significantly alter OER curves. a) Variation in OER for a dose of $15 \mathrm{~Gy}$ delivered at different dose rates, for different initial oxygen levels. At $0.1 \mathrm{~Gy} / \mathrm{s}$, a standard OER curve is observed, but as the dose rate is increased reductions in OER are seen. b) Variation in OER at a dose rate of $15 \mathrm{~Gy} / \mathrm{s}$ for different delivered doses. Again, at low doses a conventional OER curve is observed, but at higher doses the OER curves are shifted to the right as more oxygen is depleted during the exposure. In both scenarios, sparing is greatest at intermediate oxygen levels. 

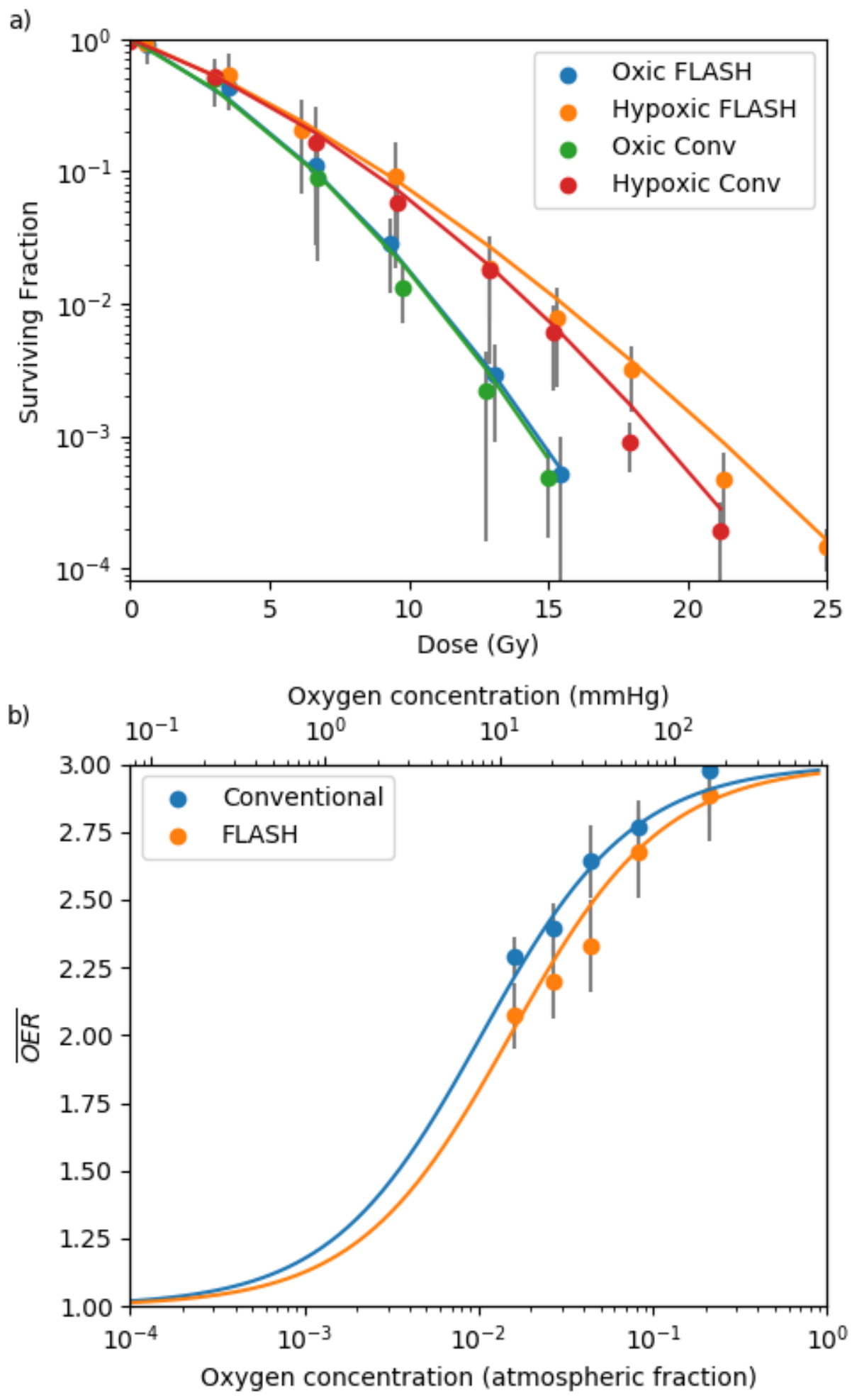

Figure 3: Oxygen depletion can effectively model FLASH effects in vitro. a) Survival of cells as a function of dose, at Conventional ( $14 \mathrm{~Gy} / \mathrm{min})$ and FLASH $(600 \mathrm{~Gy} / \mathrm{s})$ dose rates, in oxic and hypoxic $\left(1.6 \% \mathrm{O}_{2}\right.$, $12 \mathrm{mmHg}$ ) conditions. Under oxic conditions there is no significant impact of irradiation at FLASH dose rates, while under hypoxia significant sparing begins to be seen as dose increases above $10 \mathrm{~Gy}$. b) Observed OER as a function of environmental oxygen levels, for cells exposed to $18 \mathrm{~Gy}$ at normal and FLASH dose rates. Points are experimental data, while lines are model fits as described in the text. 


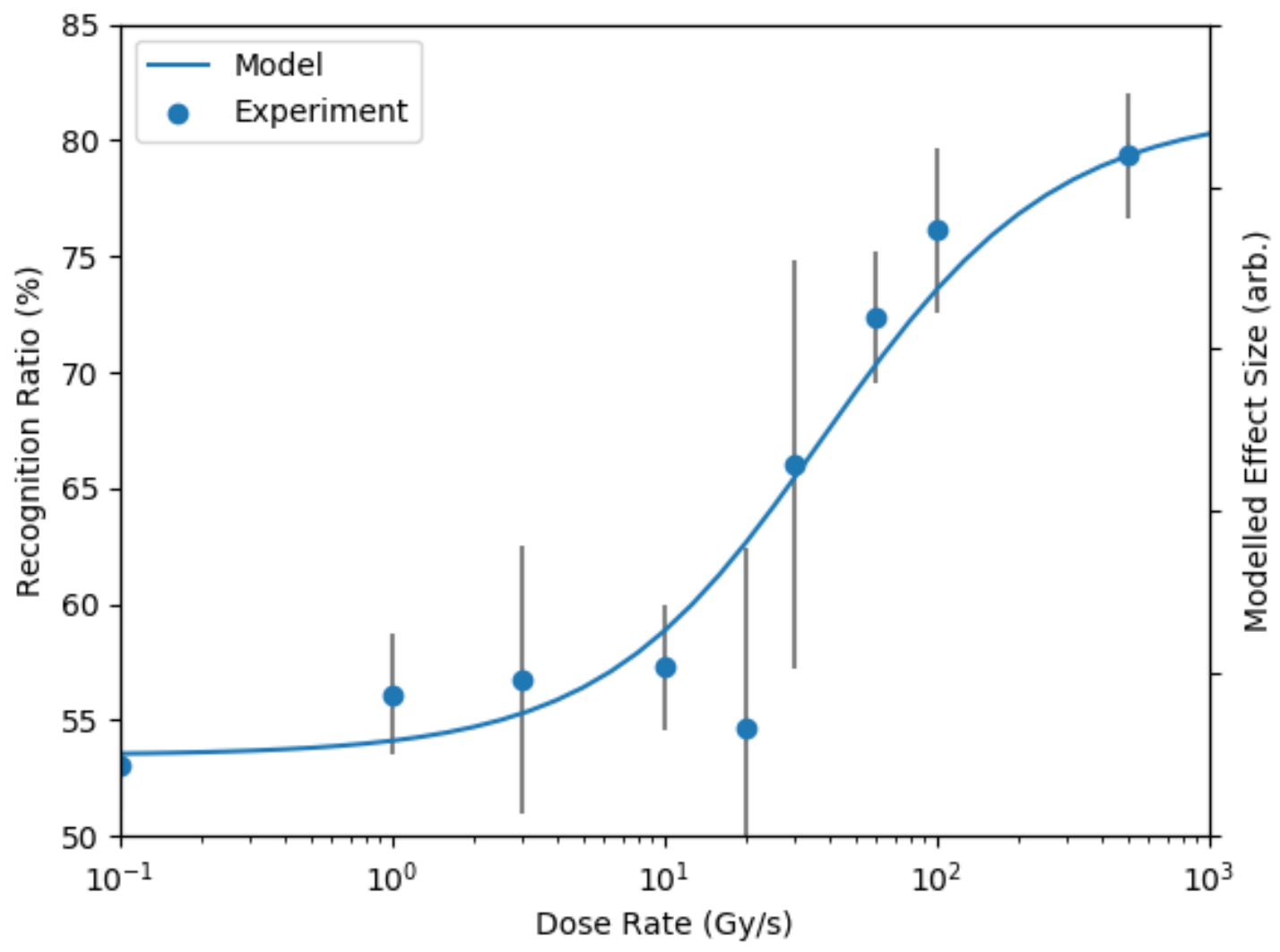

Figure 4: Oxygen depletion can also describe response kinetics in vivo. Here, data describing changes in recognition ratio in the novel object recognition test (points (2)) following exposure to 10 Gy were compared to the change in modelled effect size predicted by scaling the delivered dose by the timevarying $\overline{O E R}$ (lines), which accurately reproduced the overall trend in response. The best-fitting oxygen recovery rate was found to be $\lambda=7.9 \pm 4 \mathrm{~s}^{-1}$. 


\section{A quantitative analysis of the role of oxygen tension in FLASH radiotherapy}

\section{Supplementary Information}

\section{Alternative OER Model}

In the main text, we have simulated FLASH oxygen depletion assuming that oxygen is removed at a rate proportional to both the current oxygen level and the delivered dose rate. However, alternative models are also possible. For example, some experimental data suggests that in isolated aqueous solutions, oxygen is depleted at a roughly constant rate with delivered dose, that is:

$$
\frac{d O}{d t}=-g \dot{D}
$$

Here, $g$ takes units of the form Moles/L/Gy or ( equivalently \%/Gy or mmHg/Gy, depending on chosen units). If we assume the same model of oxygen recovery as used in the main text, then we can describe the total oxygen kinetics as:

$$
\frac{d O}{d t}=-g \dot{D}+\lambda\left(O_{e n v}-O\right)
$$

Which can be solved to give a time-varying concentration of:

$$
O=O_{e n v}-\frac{g \dot{D}}{\lambda}\left(\mathrm{e}^{-\lambda t}-1\right)
$$

As before, this assumes an initial concentration of $O_{e n v}$, which is depleted during the radiation exposure, to a minimum concentration of $O_{e n v}-g \dot{D} / \lambda$. Notably, in this model it is possible that this minimum concentration is less than $0 \%$ (that is, $O_{e n v}<g \dot{D} / \lambda$ ). This is obviously unphysical, so oxygen concentration is floored at $0 \%$ when carrying out these simulations.

This oxygen concentration prediction can be combined with the OER model as in the main text to provide an expression for the corresponding time-evolution of the OER, that is:

$$
\overline{O E R}=3-\frac{2 K}{T\left(\lambda\left(K+O_{e n v}\right)-\dot{D} g\right)}\left(\lambda T+\log \left(1+\frac{\dot{D} g\left(1-e^{-\lambda T}\right)}{\lambda\left(K+O_{e n v}\right)}\right)\right)
$$

As for the linear oxygen depletion model, this reduces to the standard OER expression in the case of either low doses or dose rates ( $\dot{D} g$ or $T$ small), but falls with increasing dose delivery as oxygen is depleted. As above, a significant difference compared to the model in the main text is that the oxygen concentration can reach zero, at which point the OER becomes a constant value of 1 . To take this into account, if the oxygen value reaches zero during an exposure, the total OER should be calculated as:

$$
\overline{O E R}=\frac{T_{\text {cross }} \overline{O E R}\left(T_{\text {cross }}\right)+\left(T-T_{\text {cross }}\right)}{T}
$$

Where $\overline{O E R}\left(T_{\text {cross }}\right)$ is the average OER to the time when the oxygen concentration reaches $0 \%$ $\left(T_{\text {cross }}\right)$, and $T$ the total exposure time. $T_{\text {cross }}$ can be calculated as:

$$
T_{\text {cross }}=-\log \left(1-\frac{O_{e n v} \lambda}{\dot{D} g}\right) / \lambda
$$




\section{Alternative OER model results}

Figures S1 to S4 present results corresponding to figures in the main text, showing the kinetics of oxygen and OER under irradiation in this model. Overall, it can be seen that similar trends are predicted, although there are some quantitative differences between different model assumptions.

As in the main text, Figure S1 begins from an oxygen level of $5 \%$ of atmospheric concentration (38 $\mathrm{mmHg}$ ), exposed to dose rates ranging from 0.1 to $100 \mathrm{~Gy} / \mathrm{s}$, with $g=0.05 \% / \mathrm{Gy}(0.38 \mathrm{mmHg} / \mathrm{Gy})$ and $\lambda=1 \mathrm{~s}^{-1}$. It shows that in this model the onset of oxygen depletion is relatively sharper with increasing dose rate, with low to moderate dose rates not impacting on oxygen availability to any measurable degree, but rapidly shifting to complete depletion between 10 and $100 \mathrm{~Gy} / \mathrm{s}$. However, based on this depletion rate from experimental data, this model suggests that the absolute rate of oxygen depletion as a function of dose is slower, requiring nearly $100 \mathrm{~Gy}$ to fully deplete $\mathrm{O}_{2}$.

Figure $\mathrm{S} 2$ shows the dependence of $\overline{O E R}$ on $\mathrm{O}_{2}$ concentration, dose- and dose rate. As in the main text, it predicts no effect at the highest or lowest $\mathrm{O}_{2}$ concentrations, but the kinetics at intermediate concentrations are quite different. In particular, rather than having a roughly equal separation for a given dose and dose rate at intermediate $\mathrm{O}_{2}$ concentrations, this model of oxygen depletion suggests curves increase in separation until quite low $\mathrm{O} 2$ concentrations, which would suggest the greatest effects are seen in highly hypoxic cells, which may not be compatible with some experimental observations. However, the exact degree of this sensitisation remains dependent on assumed model parameters.

Once again, fitting to experimental data provides some insight into these parameters, as shown in Figure S3. Here, the alternative model also effectively reproduces dose response curves for a range of conditions, showing the onset of the FLASH effect in hypoxic conditions. The best-fitting oxygen depletion rate to this data is $g=0.052 \pm 0.008 \% / G y(0.4 \mathrm{mmHg} / \mathrm{Gy})$. Interestingly, this depletion rate is in agreement with previous studies of irradiation in media, where rates between 0.043 and $0.055 \% / G y$ ( 0.33 to $0.42 \mathrm{mmHg} / \mathrm{Gy}$ ) have been reported $(14,16)$. However, this model does seem to under-estimate the observed degree of separation between the normal and FLASH irradiations at higher oxygen tensions (Figure S3b), but due to statistical uncertainties in the data this is not sufficient to clearly distinguish between the two models.

Finally, Figure S4 shows that, although this oxygen depletion model predicts a somewhat steeper onset of the FLASH effect with increasing dose rate, this is still broadly compatible with the observed response in in vivo models of brain function, although the best-fitting oxygen recovery rate is similar to that in the other model $\left(\lambda=7 \pm 4 \mathrm{~s}^{-1}\right)$.

Together, these results indicate that while there are quantitative differences between these different oxygen depletion models, the key observations about the dependence of the FLASH effect on dose, dose rate and oxygenation status remain unchanged. Within the uncertainties in the available datasets, it is not possible to statistically distinguish between these models at present. Further experimental data, mapping out this parameter space in more detail, is needed to distinguish between these and other possible models of oxygen depletion in FLASH radiotherapy. 


\section{Pulsed vs continuous exposure}

In the applications presented in the main text, we have focused on exposures to continuous dose rates, as this facilitates the efficient analytic solution of the oxygen depletion and radiation protection equations presented in the main text.

However, in practice, most FLASH exposures are delivered in a series of pulses (often lasting only a few $\mu \mathrm{s}$ ), separated by relatively long inter-pulse spacing (often many $\mathrm{ms}$ ). This will lead to some differences in the temporal structure of the oxygen depletion by radiation, but is not expected to significantly impact on the overall kinetics, assuming the pulse spacing is short compared to the kinetics of oxygen recovery.

To demonstrate this, we numerically simulated oxygen kinetics following a series of pulsed exposures at $100 \mathrm{~Gy} / \mathrm{s}, 30 \mathrm{~Gy} / \mathrm{s}$ and $10 \mathrm{~Gy} / \mathrm{s}$. In each case, $1 \mu \mathrm{s}$ radiation pulses were simulated, with $10 \mathrm{~ms}$ interpulse spacing. The dose per pulse was set to $1,0.3$ and $0.1 \mathrm{~Gy}$ to achieve the desired dose rate. This simulation made use of the first-order oxygen depletion model presented in the main text, with model parameters fixed to the same values as used in Figure 1.

As can be seen in Figure S5, the move to pulsed irradiation introduces some temporal structure at the very beginning of the exposure, where the irradiation time and pulse spacing are similar. However these differences rapidly diminish as the exposure time increases. In particular, when comparing exposures to a given total dose, there is no clear temporal structure, and the total degree of oxygen depletion is almost indistinguishable from that predicted by the continuous exposure model.

This shows that the average dose rate is a good surrogate for oxygen depletion, provided the pulse spacing is not similar in size compared to the total irradiation time e.g. when delivering only one or two pulses. 


\section{Supplementary Figures}

a)

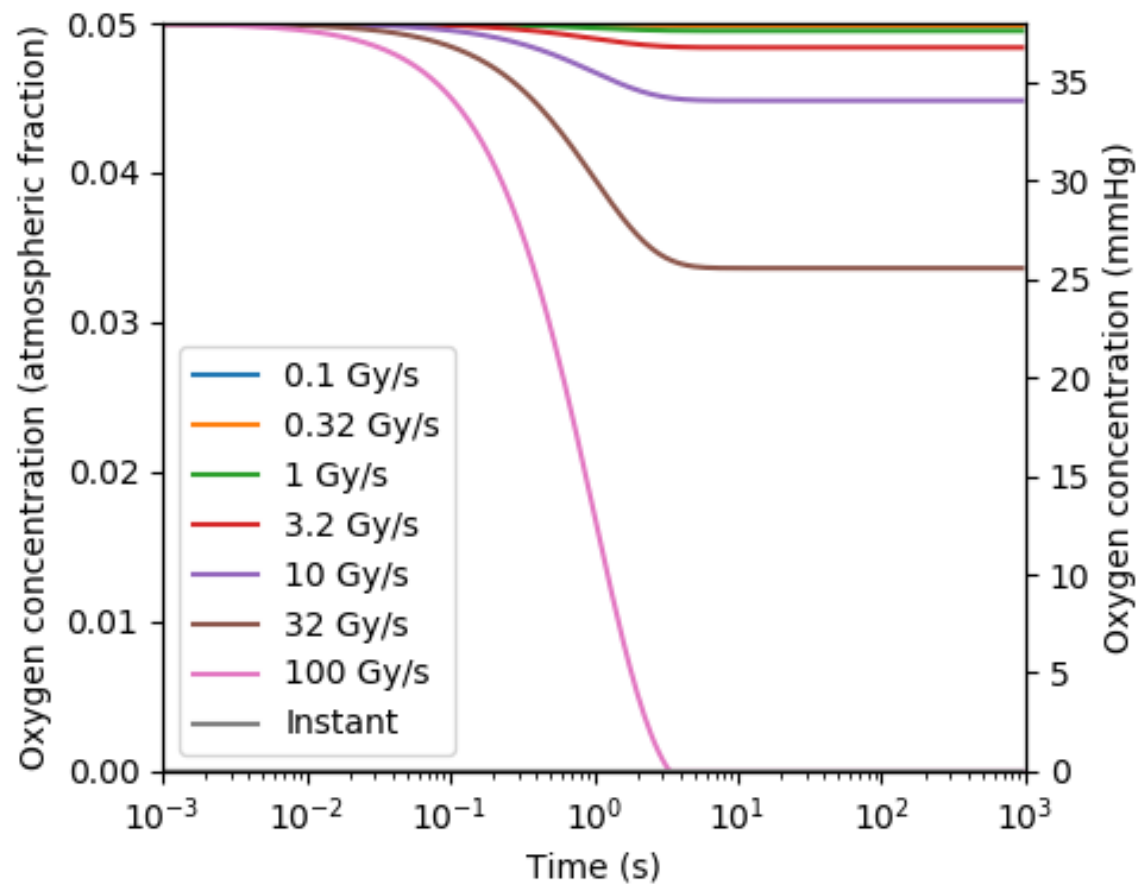

b)

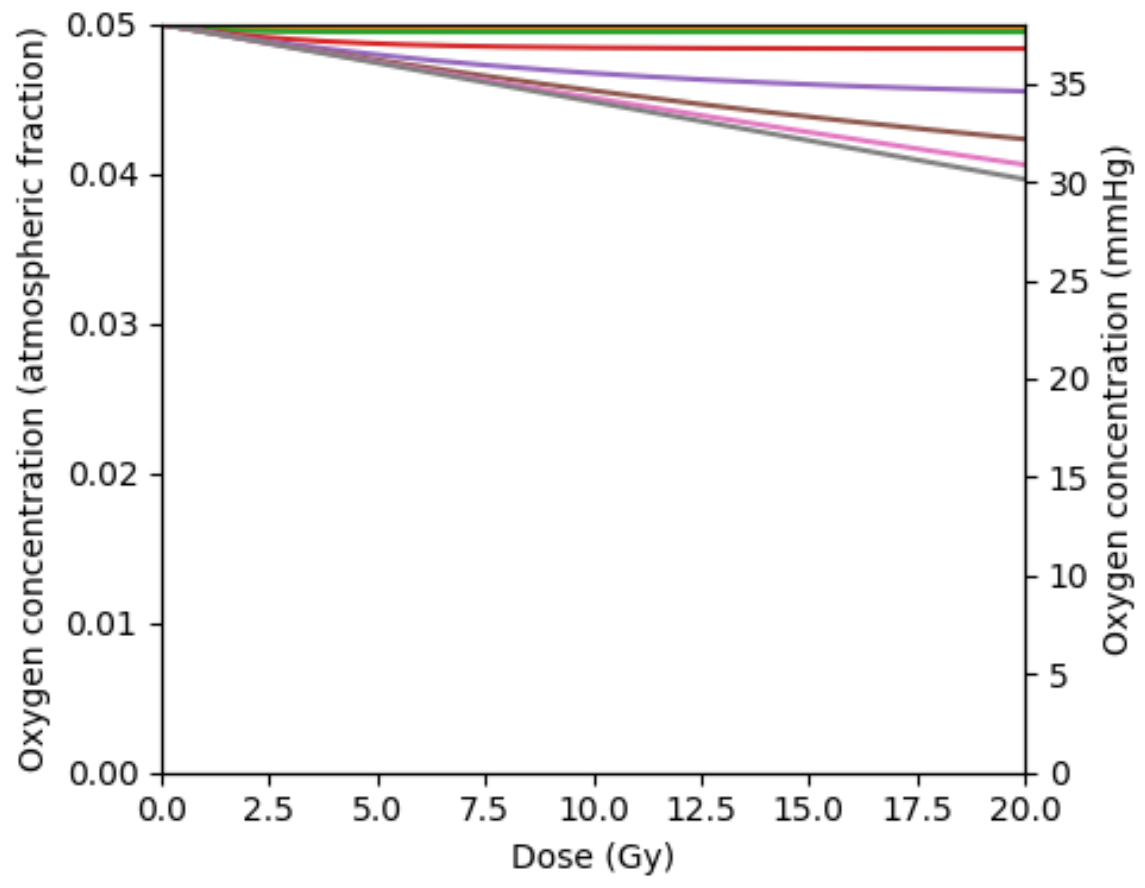

Figure S1: Oxygen depletion kinetics with constant depletion rate. As in the main text, high dose rate radiation exposures can deplete available oxygen levels. In this case, the dependence on dose rate is stronger than when depletion is proportional to oxygen concentration, potentially depleting all oxygen at sufficiently high doses and dose rates. 
a)

\section{Oxygen concentration $(\mathrm{mmHg})$}

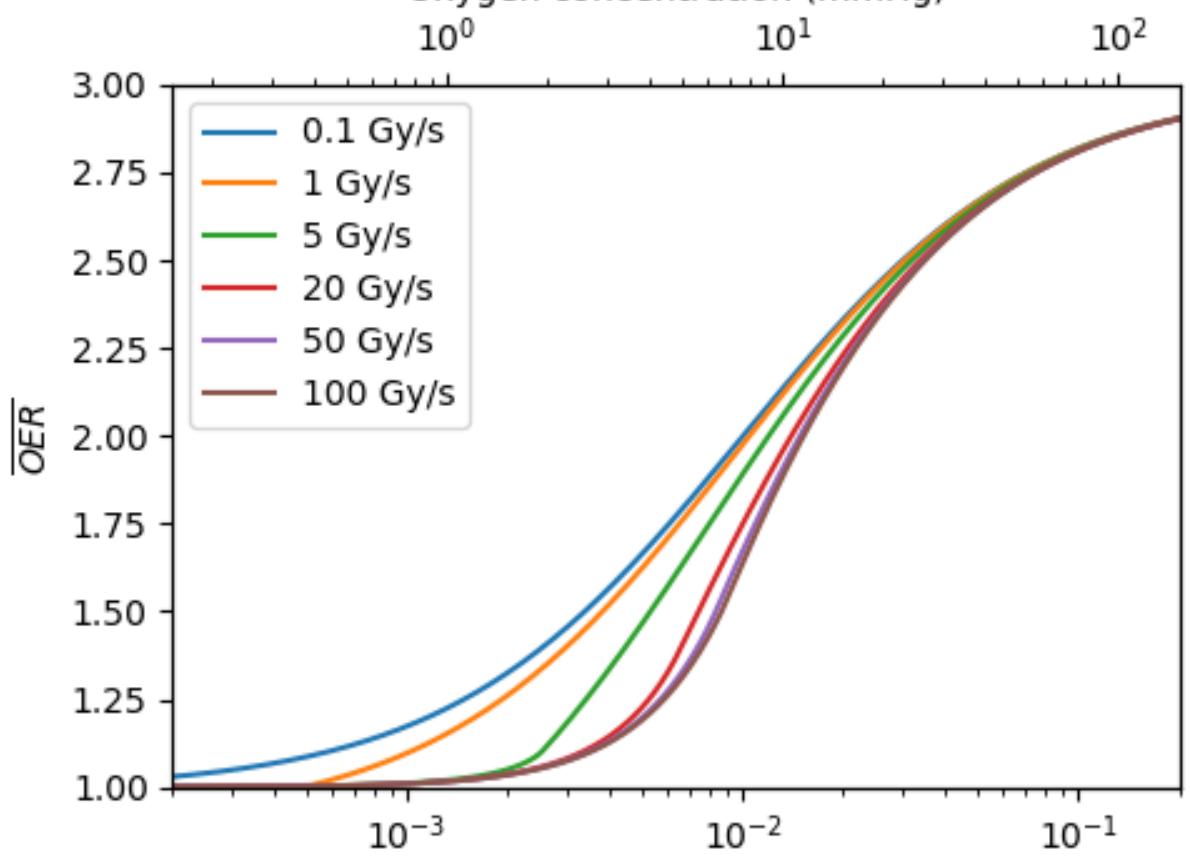

b)

$\mathrm{O}_{2}$ Concentration (atmospheric fraction)

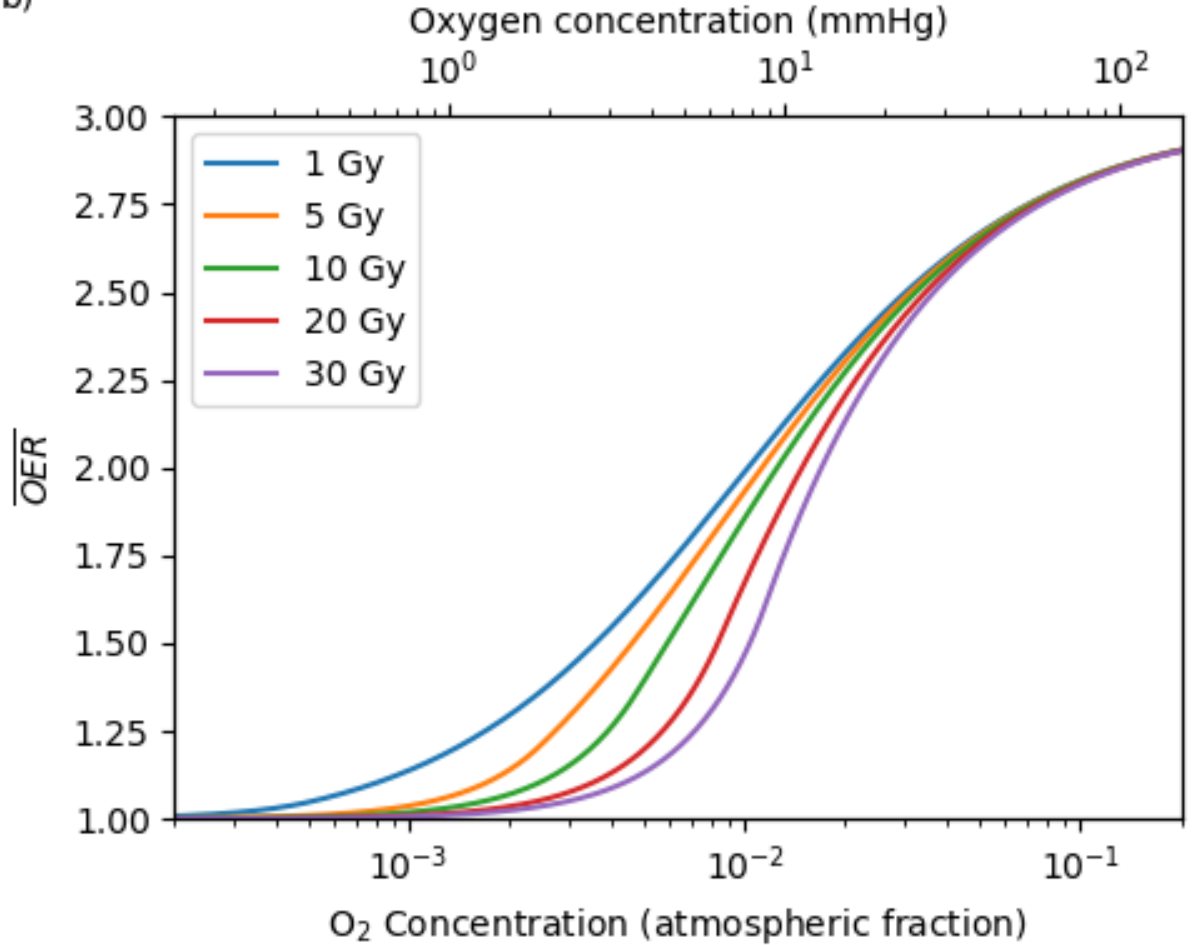

Figure S2: Alternative oxygen depletion models also suggest significant OER alterations. However, while overall trends are again qualitatively similar to the main text, with no effect at very high or very low oxygen tensions, the behaviour at intermediate concentrations is different, showing potentially greatest separation at lower oxygen levels (at $<1 \% \mathrm{O}_{2}, 7 \mathrm{mmHg}$ ). 

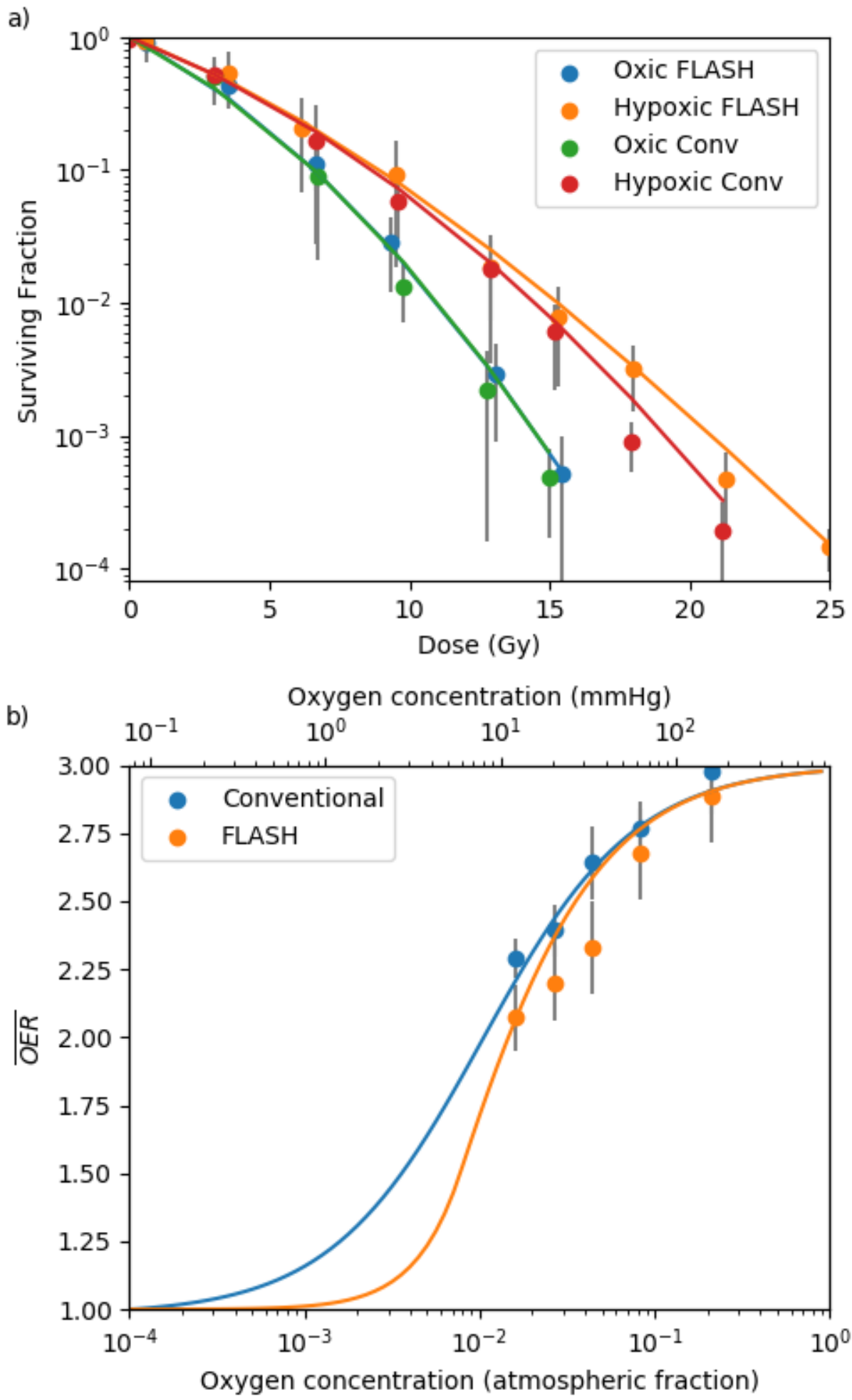

Figure S3: Alternative oxygen depletion models can also effectively reproduce experimental observations. Dose dependence is well-reproduced as in main text (top). Fit as a function of varying oxygen concentration shows somewhat reduced separation compared to experimental data (bottom), however this deviation is not statistically significant. 


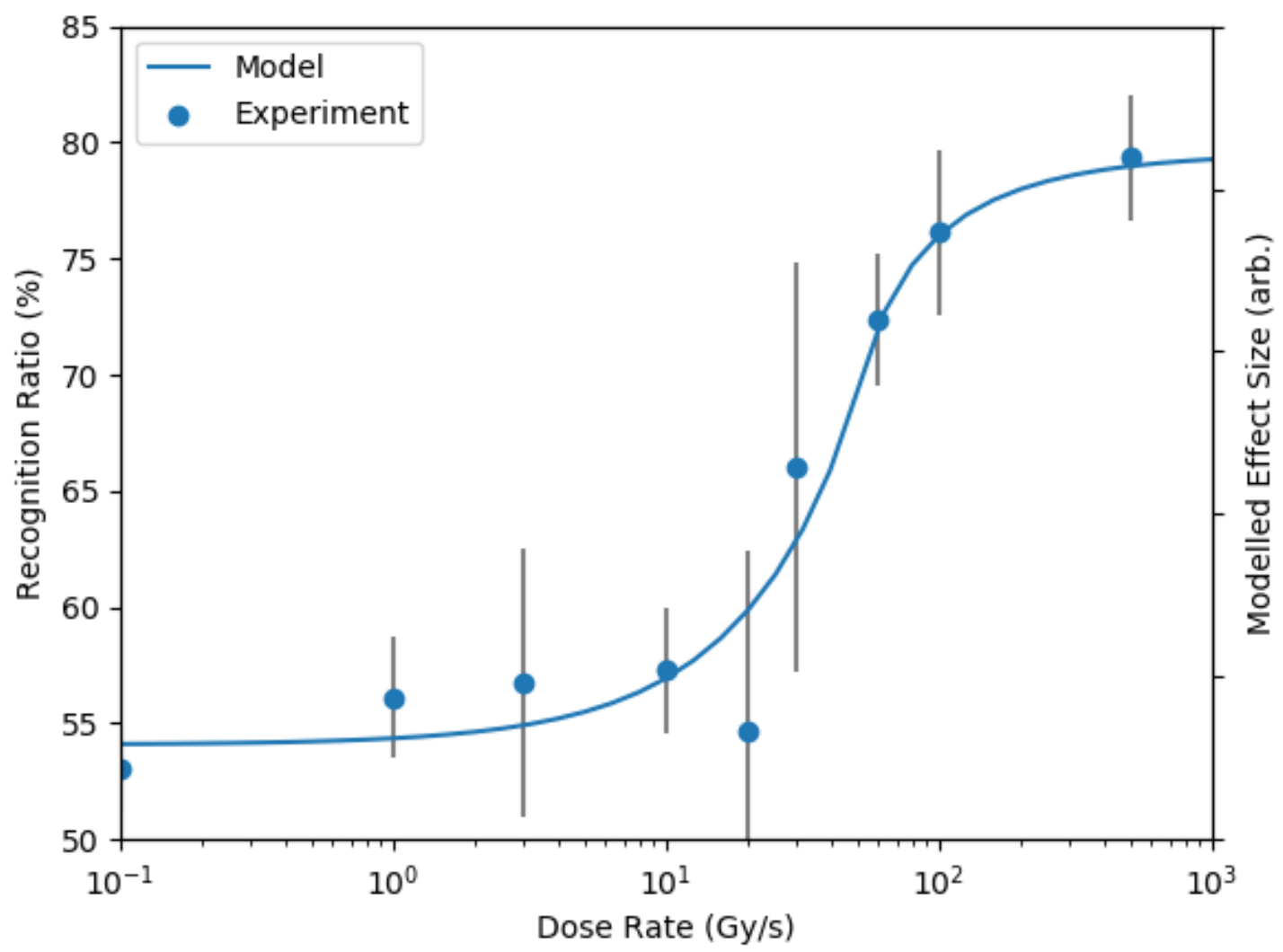

Figure S4: Kinetics of oxygen depletion can also be similarly reproduced by this model. Here, a similar oxygen recovery rate provides the best fit $\left(\lambda=7 \pm 4 \mathrm{~s}^{-1}\right)$, with a slightly steeper dose rate dependence, but the overall trends remain similar. 

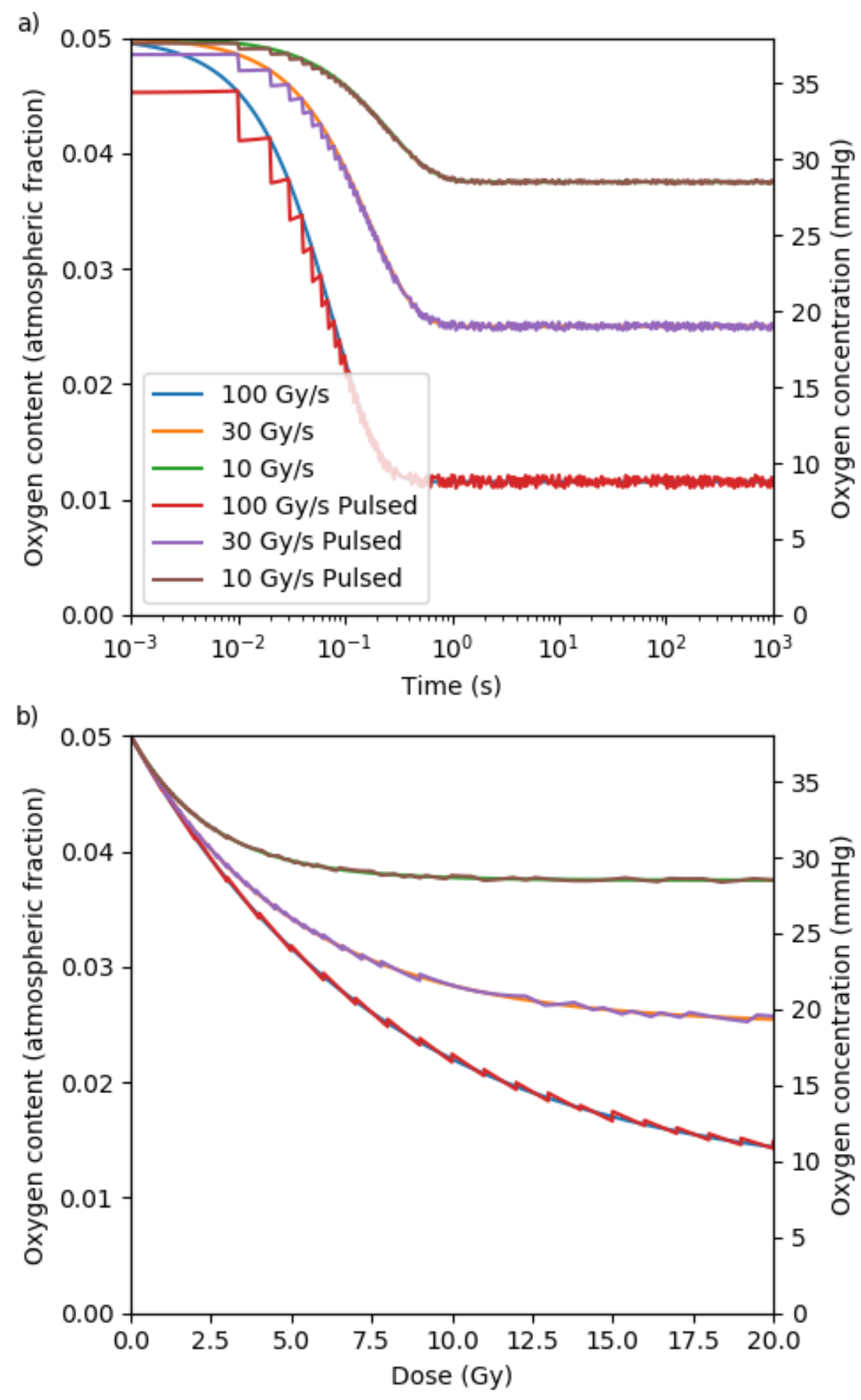

Figure S5: Oxygen depletion kinetics for pulsed and continuous exposure. Oxygen depletion was modelled using either continuous exposures or pulsed exposures ( $1 \mu \mathrm{s}$ pulses, $10 \mathrm{~ms}$ spacing) at a range of dose rates. While some differences are observed at initial timepoints when the inter-pulse spacing is similar to the total exposure time, it can be seen that for any given delivered dose (bottom), the continuous exposure model very closely approximates the degree of oxygen depletion in the pulsed exposure. 